\title{
Asymptotic Exactness of Magnetic Thomas-Fermi Theory at Nonzero Temperature
}

\author{
Bergthór Hauksson $^{1 *}$ and Jakob Yngvason ${ }^{2}$ \\ 1. Kögun hf, Lynghálsi 9, IS-110 Reykjavik, Iceland \\ 2. Institut für Theoretische Physik, Universität Wien, \\ Boltzmanngasse 5, A-1090 Vienna, Austria \\ Dedicated to Elliott H. Lieb on the occasion of his 70th birthday
}

\begin{abstract}
We consider the grand canonical pressure for Coulombic matter with nuclear charges $\sim Z$ in a magnetic field $B$ and at nonzero temperature. We prove that its asymptotic limit as $Z \rightarrow \infty$ with $B / Z^{3} \rightarrow 0$ can be obtained by minimizing a Thomas-Fermi type pressure functional.
\end{abstract}

\section{Introduction}

This paper intends to add one more chapter to the saga of rigorous Thomas-Fermi theory in which Elliott H. Lieb played a prominent role [1]. The issue is the derivation of Thomas Fermi theory at nonzero temperature in a strong magnetic field from quantum statistical mechanics. The asymptotic exactness of Thomas-Fermi theory for Coulombic matter in its ground state was first proved by Lieb and Simon in the fundamental paper 2]. A shorter proof, using coherent states, was given by Lieb in [3] and several ideas in the present paper were inspired by that proof.

Thomas-Fermi Theory for matter in extremely strong magnetic fields is important for the physics of neutron stars, cf. [4, 5, 6] and references quoted therein. This theory was analyzed from the point of view of mathematical physics in [7, 8, 9] and its status as a limit of quantum mechanics in a certain parameter range firmly established; an extension of the asymptotics to inhomogeneous magnetic fields is in [10. All these works are concerned with the ground state, but non-magnetic TF theory at temperatures $T>0$ has been treated in 11, 12, 13. Magnetic TF theory at nonzero temperature was studied in 14 and applied to the equation of state for matter in the crust of a neutron star, but a proof of its asymptotic exactness has,

* Work partially supported by the Research Fund of the University of Iceland.

(c) 2003 by the authors. This paper may be reproduced, in its entirety, for non-commercial purposes. 
to the best of our knowledge, not been published until now. The proof we give here brings together techniques from [11, 12] and [7, 8, with several amendments and additions.

We start our discussion with some heuristic considerations. A possible point of departure for a motivation of TF theory, both at $T=0$ and $T>0$, is the thermodynamic relation between the particle density $\rho$, the chemical potential $\mu$, and the pressure $P$ for a homogeneous gas of noninteracting particles (electrons):

$$
\rho=\partial P(\mu) / \partial \mu=: P^{\prime}(\mu) .
$$

The next step is to consider electrons that interact with with each other via Coulomb forces and also with an external potential $V$ (arising from nuclei in fixed positions as well as a confining potential that prevents the electrons from escaping to infinity). The electron density now depends on the position $\mathbf{x}$. The TF theory is formally obtained from (1.1) by replacing the constant density $\rho$ by a position dependent density $\rho(\mathbf{x}) \geq 0$ and the chemical potential by a position dependent chemical potential $\mu(\mathbf{x})$, imposing as an equilibrium condition that the total electrochemical potential

$$
\mu_{\mathrm{TF}}=\mu(\mathbf{x})+V_{\rho}(\mathbf{x})
$$

with

$$
V_{\rho}(\mathbf{x})=V(\mathbf{x})+\rho *|\mathbf{x}|^{-1},
$$

should be independent of $\mathbf{x}$. The result is the Thomas-Fermi equation:

$$
\rho(\mathbf{x})=P^{\prime}\left(\mu_{\mathrm{TF}}-V_{\rho}(\mathbf{x})\right) .
$$

For given $P, V$ and $\mu_{\mathrm{TF}}$ this is a nonlinear integral equation for $\rho(\mathbf{x})$. The total particle number is

$$
\int \rho(\mathbf{x}) d \mathbf{x}=N
$$

The equation (1.4) is the variational equation associated with the minimization problem for the TF pressure functional of the density, ${ }^{*}$

$$
\mathcal{P}[\rho]=\int P\left(\mu_{\mathrm{TF}}-V_{\rho(\mathbf{x})}\right) d \mathbf{x}+D(\rho, \rho)
$$

with

$$
D(\rho, \rho)=\frac{1}{2} \iint \frac{\rho(\mathbf{x}) \rho\left(\mathbf{x}^{\prime}\right)}{\left|\mathbf{x}-\mathbf{x}^{\prime}\right|} d \mathbf{x} d \mathbf{x}^{\prime}
$$

The minimum of (1.6) over all nonnegative functions $\rho$ will be called the TF pressure.

\footnotetext{
${ }^{*}$ Instead of considering $\mathcal{P}$ as a functional of the density, it could equivalently be considered as a functional of the potential $V_{\rho}$. Note that $\rho$ and hence $D(\rho, \rho)$ is determined by $V_{\rho}$ because $4 \pi \rho(\mathbf{x})=-\Delta \rho *|\mathbf{x}|^{-1}=\Delta\left(V-V_{\rho}(\mathbf{x})\right)$. While this point of view (which is related to that of Firsov [15] in standard TF theory) may be more natural if $\mathcal{P}$ is regarded as a Legendre transformation of the free energy functional $\mathcal{F}(1.10)$, we find it convenient in the present context to regard $\mathcal{P}$ as a functional of $\rho$.
} 
An alternative form of the TF equation is obtained if one replaces $P$ by its Legendre transform, the free energy density

$$
f(\rho)=\sup _{\mu}\{\mu \rho-P(\mu)\} .
$$

From (1.8) it follows that $\partial f / \partial \rho=: f^{\prime}$ is the inverse of $P^{\prime}$. Hence the TF equation (1.4) can also be written

$$
\left.f^{\prime}(\rho(\mathbf{x}))+V_{\rho}(\mathbf{x})\right)=\mu_{\mathrm{TF}} .
$$

This form of the TF equation is associated with the minimization problem for the free energy functional

$$
\left.\mathcal{F}[\rho]=\int\{f(\rho(\mathbf{x}))+V(\mathbf{x})) \rho(\mathbf{x})\right\} d \mathbf{x}+D(\rho, \rho)
$$

with (1.5) as a subsidiary condition and $\mu_{\mathrm{TF}}$ as a Lagrange multiplier.

In the sequel we shall investigate the functional (1.6). Our main result is its asymptotic exactness in the case of electrons in a constant magnetic field and at nonzero temperature. This amounts to taking a semiclassical limit of the grand canonical pressure for the quantum mechanical many-body problem. The corresponding problem for the free energy, i.e., the canonical partition function, is technically more involved and will not be tackled here.

We now introduce some notation that will allow us to state the results precisely.

The many-body Hamiltonian considered in this paper is

$$
\left.H_{N, Z, B}=\sum_{i=1}^{N}\left\{\left[\mathbf{p}_{i}+\mathbf{A}\left(\mathbf{x}_{i}\right)\right) \cdot \boldsymbol{\sigma}_{i}\right]^{2}+V_{Z, B}\left(\mathbf{x}_{i}\right)\right\}+\sum_{1 \leq i<j \leq N}\left|\mathbf{x}_{i}-\mathbf{x}_{j}\right|^{-1}
$$

Here $\mathbf{p}=-\mathrm{i} \nabla, \mathbf{A}(\mathbf{x})=\frac{1}{2}\left(-B x_{2}, B x_{1}, 0\right)$ is the vector potential of a homogeneous magnetic field of strength $B$ in the $x_{3}$-direction, and $\boldsymbol{\sigma}$ is the vector of Pauli matrices. Atomic units are chosen so that $\hbar=2 m=e=1$, and the temperature unit is such that the Boltzmann constant $k$ is also 1 . The external potential is

$$
V_{Z, B}(\mathbf{x})=-Z \sum_{k=1}^{K} \frac{z_{k}}{\left|\mathbf{x}-\ell \mathbf{X}_{k}\right|}+Z \ell^{-1} W\left(\ell^{-1} \mathbf{x}\right)
$$

where $W$ is a confining potential that tends sufficiently rapidly to $\infty$ for $|\mathbf{x}| \rightarrow \infty$ so that $\exp (-W(\mathbf{x}) / T)$ is integrable for all $T>0$. It will also be assumed to satisfy some regularity conditions stated later. The $\mathbf{X}_{k}$ are fixed positions of nuclei with fixed charges $z_{k} \leq 1$ which are scaled by an overall parameter $Z$. The length scaling factor

$$
\ell=\ell_{Z, B}=Z^{-1 / 3}\left[1+\left(B / Z^{4 / 3}\right)\right]^{-2 / 5}
$$

is the one appropriate for TF atoms in a magnetic field, cf. [8]. 
The Hamiltonian $H_{N, Z, B}$ operates on the $N$-electron Hilbert space of antisymmetric wave functions in space and spin variables:

$$
\mathcal{H}_{N}=\wedge^{N} L^{2}\left(\mathbb{R}^{3}, \mathbb{C}^{2}\right)
$$

The corresponding Fock space is

$$
\widehat{\mathcal{H}}=\bigoplus_{N=0}^{\infty} \mathcal{H}_{N}
$$

with $\mathcal{H}_{0}=\mathbb{C}$. If $A_{N}$ are operators on $\mathcal{H}_{N}, N=0,1, \ldots$, we denote the operator $\oplus_{N=0}^{\infty} A_{N}$ on $\widehat{\mathcal{H}}$ by $\widehat{A}$. In particular,

$$
\widehat{H}_{Z, B}=\bigoplus_{N=0}^{\infty} H_{N, Z, B}
$$

The grand canonical pressure at chemical potential $\mu$ and temperature $T$ is*

$$
P^{\mathrm{QM}}(\mu, B, T, Z)=T \ln \operatorname{tr} \exp \left[-\left(\widehat{H}_{Z, B}-\mu \widehat{N}\right) / T\right] .
$$

The free 1-particle Hamiltonian

$$
H_{0}=[(\mathbf{p}+\mathbf{A}(\mathbf{x})) \cdot \boldsymbol{\sigma}]^{2}
$$

has the Landau spectrum

$$
\varepsilon_{\nu}(p)=2 B \nu+p^{2}, \quad \nu=0,1, \ldots, p \in \mathbb{R}
$$

with degeneracy (pro unit area in the $\left(x_{1}, x_{2}\right)$-plane)

$$
d_{\nu}(B)=\left\{\begin{array}{cl}
B /(2 \pi) & \text { if } \quad \nu=0 \\
B / \pi & \text { if } \quad \nu \geq 1
\end{array}\right.
$$

The pressure of a free electron gas at temperature $T$ in a magnetic field and with chemical potential $\mu$ is

$$
P_{T, B}(\mu)=T \sum_{\nu=0}^{\infty} d_{\nu}(B) \int_{-\infty}^{\infty} \ln \left[1+\exp \left\{-\left(\varepsilon_{\nu}(p)-\mu\right) / T\right\}\right] d p .
$$

The magnetic Thomas-Fermi pressure functional is obtained by taking $P$ in (1.6) to be (1.201):

$$
\mathcal{P}^{\mathrm{MTF}}[\rho ; Z, \mu, T, B]=\int P_{T, B}\left(\mu-V_{Z, B, \rho}(\mathbf{x})\right) d^{3} \mathbf{x}+D(\rho, \rho)
$$

with

$$
V_{Z, B, \rho}(\mathbf{x})=V_{Z, B}(\mathbf{x})+\rho *|\mathbf{x}|^{-1} .
$$

The pressure according to MTF theory is

$$
P^{\mathrm{MTF}}(Z, \mu, T, B):=\inf _{\rho \geq 0} \mathcal{P}^{\mathrm{MTF}}[\rho ; \mu, T, B, Z] .
$$

We can now state our main result:

\footnotetext{
${ }^{*}$ Note that $\mu$ denotes here the total electrochemical potential and not the chemical potential of
} the free electron gas denoted by the same letter in in (1.1) and (1.20). 
Theorem 1.1 (The MTF pressure is a limit of the QM pressure). If $Z, \mu, T \rightarrow \infty$ with $\mu /\left(Z \ell_{B, Z}^{-1}\right)$ and $T /\left(Z \ell_{B, Z}^{-1}\right)$ fixed but $B / Z^{3} \rightarrow 0$, where $\ell_{B, Z}$ is given by (1.13), then

$$
\frac{P^{\mathrm{QM}}(\mu, T, B, Z)}{P^{\mathrm{MTF}}(\mu, T, B, Z)} \rightarrow 1
$$

The main steps in the proof are as follows. In the next section we discuss the MTF functional in more detail, in particular the existence and uniqueness of a minimizer and its properties. In Section 3 we consider a Hamiltonian with a mean field and the corresponding pressure functional. Section 4 contains the basic semiclassical limit theorem, which is proved using the magnetic coherent states introduced in [7] and [8]. As a corollary one obtains the convergence of the mean field pressure to the MTF pressure. Here it is important that the error bounds in the semiclassical theorem are uniform in the relevant parameters. In the last section the proof of Theorem 1.1 is completed by estimating the many-body pressure in terms of the mean field pressure.

\section{The MTF pressure functional}

In this section we collect some basic facts about the pressure functional (1.21). We start with some formulas and estimates for the pressure (1.20) of the free electron gas in a magnetic field. It can also be written as

$$
P_{T, B}(\mu)=\int_{0}^{\infty} \frac{G(\varepsilon)}{\exp \{(\varepsilon-\mu) / T\}+1} d \varepsilon
$$

with the integrated density of states

$$
G(\varepsilon)=\frac{B}{2^{1 / 2} \pi^{2}}\left[\varepsilon^{1 / 2}+2 \sum_{\nu=0}^{\infty}|\varepsilon-2 B \nu|_{+}^{1 / 2}\right] .
$$

Here $|s|_{+}:=|s|$ for $s \geq 0$ and 0 otherwise. The corresponding formulas for $P_{T, B}^{\prime}=$ $\partial P_{T, B} / \partial \mu$ are

$$
\begin{aligned}
P_{T, B}^{\prime}(\mu) & =\sum_{\nu=0}^{\infty} d_{\nu}(B) \int_{-\infty}^{\infty} \frac{1}{\exp \left\{\left(\varepsilon_{\nu}(p)-\mu\right) / T\right\}+1} d p \\
& =\int_{0}^{\infty} \frac{G^{\prime}(\varepsilon)}{\exp \{(\varepsilon-\mu) / T\}+1} d \varepsilon .
\end{aligned}
$$

The scaling properties of $P_{T, B}$ can be seen by writing

$$
P_{T, B}(\mu)=\frac{B T^{3 / 2}}{2^{1 / 2} \pi^{2}}\left[I_{1 / 2}(\mu / T)+2 \sum_{\nu=1}^{\infty} I_{1 / 2}(\mu-(2 B \nu / T))\right],
$$

and

$$
P_{T, B}^{\prime}(\mu)=\frac{B T^{1 / 2}}{2^{3 / 2} \pi^{2}}\left[I_{-1 / 2}(\mu / T)+2 \sum_{\nu=1}^{\infty} I_{-1 / 2}(\mu-(2 B \nu / T))\right]
$$


with

$$
I_{k}(x)=\int_{0}^{\infty} \frac{y^{k}}{e^{y-x}+1} d y .
$$

Using the inequalities $\left.\frac{1}{2} \theta(-s) \leq\left(e^{s}+1\right)^{-1} \leq \theta(-s)+e^{-|s|}\right)$ we obtain from (2.1) the following simple estimates, treating the sum over $\nu \geq 1$ as a Riemannian approximation for an integral:

$$
\begin{aligned}
c\left(B|\mu|_{+}^{3 / 2}+|\mu|_{+}^{5 / 2}\right) \leq P_{T, B}(\mu) & \\
& \leq C\left(B|\mu|_{+}^{3 / 2}+|\mu|_{+}^{5 / 2}+e^{-|\mu| / T}\left(B T^{3 / 2}+T^{5 / 2}\right)\right)
\end{aligned}
$$

with constants $c>0$ and $C<\infty$. In the same way we obtain

$$
\begin{aligned}
c^{\prime}\left(B|\mu|_{+}^{1 / 2}+|\mu|_{+}^{3 / 2}\right) \leq & P_{T, B}^{\prime}(\mu) \\
& \leq C^{\prime}\left(B|\mu|_{+}^{1 / 2}+|\mu|_{+}^{3 / 2}+e^{-|\mu| / T}\left(B T^{1 / 2}+T^{3 / 2}\right)\right) .
\end{aligned}
$$

As domain of definition for the magnetic pressure functional we take

$$
\mathcal{M}=\{\rho: \rho(\mathbf{x}) \geq 0, D(\rho, \rho)<\infty\} .
$$

To see that $\mathcal{P}^{\operatorname{MTF}}[\rho ; \mu, T, B, Z]$ is well defined and $<\infty$ for $\rho \in \mathcal{M}$ we use (2.8) together with

Lemma 2.1 (Coulomb bound). If $\rho \geq 0$ with $D(\rho, \rho)<\infty$, then the potential $v_{\rho}(\mathbf{x})=\rho *|\mathbf{x}|^{-1}$ is in $L^{6}\left(\mathbb{R}^{3}\right)$; in fact

$$
\left\|v_{\rho}\right\|_{6}^{2} \leq \text { (const.) } D(\rho, \rho) .
$$

Proof. Since $4 \pi \rho=-\nabla^{2} v_{\rho}$, we can write $D(\rho, \rho)=\left(\right.$ const.) $\left\|\nabla v_{\rho}\right\|_{2}^{2}$. We then use the Sobolev inequality in $\mathbb{R}^{3}$ [16], i.e., $\|f\|_{6} \leq$ (const.) $\|\nabla f\|_{2}$.

From this lemma follows that $\mu-V_{Z, B, \rho} \in L^{5 / 2}\left(\mathbb{R}^{3}\right)_{\text {loc }}$ and hence also in $L^{3 / 2}\left(\mathbb{R}^{3}\right)_{\text {loc }}$ for $\rho \in \mathcal{M}$. Moreover, since $W(\mathbf{x})$ tends to $\infty$ if $\mathbf{x} \rightarrow \infty$ while the negative potential from the nuclei tends to zero, there is an $R \geq 0$ such that $\left|\mu-V_{Z, B, \rho}\right|_{+}=0$ and $\left|\mu-V_{Z, B, \rho}\right| \geq Z \ell^{-1} W\left(\ell^{-1} \mathbf{x}\right)-$ (const.) for $|\mathbf{x}| \geq R$. The finiteness of $\mathcal{P}^{\mathrm{MTF}}[\rho ; \mu, T, B, Z]$ now follows from (2.8) by splitting the integration domain in (1.21) into $|\mathbf{x}| \leq R$ and $|\mathbf{x}|>R$.

The MTF pressure functional is nonnegative and strictly convex since $D(\rho, \rho)$ is strictly convex, $\rho \mapsto v_{\rho}$ is linear and $s \mapsto \ln (1+\exp (-s))$ strictly convex. Moreover, if $\mathcal{M}$ is equipped with the topology defined by the Hilbert norm $D(\rho, \rho)^{1 / 2}$, then $\mathcal{P}^{\mathrm{MTF}}$ is weakly lower semicontinuous. This can be seen by representing the convex functional as a supremum of affine, weakly continuous functionals in a similar way as in [12, (4.1.10). This continuity and strict convexity implies by standard arguments (c.f., e.g. 3]) that the functional has a unique minimizer $\rho^{\mathrm{MTF}} \in \mathcal{M}$ (depending on 
the parameters $\mu, T, B, Z)$. It is the unique solution to the $M T F$ equation, i.e., the variational equation for the minimization problem,

$$
\rho(\mathbf{x})=P_{T, B}^{\prime}\left(\mu-V_{Z, B, \rho}(\mathbf{x})\right) .
$$

Eq. (2.5) implies the following scaling property of the MTF functional:

$$
\mathcal{P}^{\mathrm{MTF}}[\rho ; \mu, T, B, Z]=Z^{2} \ell^{-1} \tilde{\mathcal{P}}^{\mathrm{MTF}}[\tilde{\rho} ; \tilde{\mu}, \tilde{T}, \beta]
$$

where

$$
\begin{gathered}
\beta=B / Z^{4 / 3}, \quad \ell=Z^{-1 / 3}(1+\beta)^{-2 / 5}, \\
\rho(\mathbf{x})=Z \ell^{-3} \tilde{\rho}\left(\ell^{-1} \mathbf{x}\right) \\
\tilde{\mu}=\mu /\left(Z \ell^{-1}\right), \quad \tilde{T}=T /\left(Z \ell^{-1}\right)
\end{gathered}
$$

and

$$
\tilde{\mathcal{P}}^{\mathrm{MTF}}[\tilde{\rho} ; \tilde{\mu}, \tilde{T}, \beta]=(1+\beta)^{-3 / 5} \int P_{\tilde{T}, \tilde{B}}\left(\tilde{\mu}-\tilde{V}_{\tilde{\rho}}(\mathbf{x})\right) d \mathbf{x}+D(\tilde{\rho}, \tilde{\rho})
$$

with

$$
\tilde{B}=B /\left(Z \ell^{-1}\right)=\beta(1+\beta)^{-2 / 5}
$$

and

$$
\tilde{V}_{\tilde{\rho}}(\mathbf{x})=-\Phi_{\mathrm{C}}(\mathbf{x})+W(\mathbf{x})+\tilde{\rho} *|\mathbf{x}|^{-1}
$$

with

$$
\Phi_{\mathrm{C}}(\mathbf{x})=\sum_{k=1}^{K} \frac{z_{k}}{\left|\mathbf{x}-\mathbf{X}_{k}\right|} .
$$

We can also include the limiting cases $\beta=0$ and $\beta=\infty$ : The case $\beta=0$ is just the temperature dependent TF without magnetic field considered in [1, 12, 13, while $\beta \rightarrow \infty$ means that only the lowest Landau level contributes. It is the $T$ dependent version of the STF theory in [8]. In fact, as is easily seen from (2.5),

$$
\lim _{\beta \rightarrow \infty}(1+\beta)^{-3 / 5} P_{\tilde{B}, \tilde{T}}(\mu)=\frac{\tilde{T}^{3 / 2}}{2^{1 / 2} \pi^{2}} I_{1 / 2}(\mu / \tilde{T})=: P_{\tilde{T}}^{\infty}(\mu)
$$

and hence

$$
\lim _{\beta \rightarrow \infty} \tilde{\mathcal{P}}^{\operatorname{MTF}}[\tilde{\rho} ; \tilde{\mu}, \tilde{T}, \beta]=\int P_{\tilde{T}}^{\infty}\left(\tilde{\mu}-\tilde{V}_{\tilde{\rho}}(\mathbf{x})\right) d \mathbf{x}+D(\tilde{\rho}, \tilde{\rho})=: \tilde{\mathcal{P}}^{\infty}[\tilde{\rho} ; \tilde{\mu}, \tilde{T}]
$$

As last topic in this section we derive some uniform bounds for the minimizing density of the MTF functional and the corresponding Coulomb potential. These bounds will be needed for the semiclassical limit theorem in Section 4 . 
Lemma 2.2 (Bounds for MTF minimizer). Let $\tilde{\rho}_{\beta}$ be the minimizer of $\tilde{\rho} \mapsto$ $\tilde{\mathcal{P}}^{\mathrm{MTF}}[\tilde{\rho} ; \tilde{\mu}, \tilde{T}, \beta]$ for fixed $\tilde{\mu}, \tilde{T}$. Then

(i) The potentials $v_{\beta}(\mathbf{x})=\tilde{\rho}_{\beta} *|\mathbf{x}|^{-1}$ are bounded in $L^{6}(\mathbb{R})$ uniformly in $\beta$.

(ii) $\tilde{\rho}_{\beta} \in L^{p}\left(\mathbb{R}^{3}\right)$ for $1 \leq p<2$ and $\left\|\tilde{\rho}_{\beta}\right\|_{p}$ is uniformly bounded in $\beta$.

(iii) Let $j_{r}=r^{-3} j(\mathbf{x} / r)$ where $0 \leq j \in C_{0}^{\infty}\left(\mathbb{R}^{3}\right)$ satisfies $\int j(\mathbf{x}) d \mathbf{x}=1$. Then for all $R<\infty$ and $p<3$

$$
\left.\left.\int_{|\mathbf{x}| \leq R}\left|v_{\beta}(\mathbf{x})-v_{\beta} *\right| \mathbf{x}\right|^{-1}\right|^{p} d \mathbf{x} \rightarrow 0
$$

uniformly in $\beta$, as $r \rightarrow 0$.

Proof. (i) This follows from Lemma 2.1 and the bound

$$
D\left(\tilde{\rho}_{\beta}, \tilde{\rho}_{\beta}\right) \leq \tilde{\mathcal{P}}^{\mathrm{MTF}}\left[\tilde{\rho}_{\beta} ; \tilde{\mu}, \tilde{T}, \beta\right] \leq \tilde{\mathcal{P}}^{\mathrm{MTF}}[0 ; \tilde{\mu}, \tilde{T}, \beta] .
$$

The right side is continuous in $\beta \geq 0$ and converges to $\tilde{\mathcal{P}}^{\infty}[0 ; \tilde{\mu}, \tilde{T}]<\infty$ for $\beta \rightarrow \infty$. Hence $D\left(\tilde{\rho}_{\beta}, \tilde{\rho}_{\beta}\right)$ is uniformly bounded in $\beta$.

(ii) The MTF equation for the scaled density is

$$
\tilde{\rho}_{\beta}(\mathbf{x})=(1+\beta)^{-3 / 5} P_{\tilde{T}, \tilde{B}}^{\prime}\left(\tilde{\mu}+\Phi_{C}(\mathbf{x})-W(\mathbf{x})-\tilde{\rho}_{\beta} *|\mathbf{x}|^{-1}\right) .
$$

Now, since $P^{\prime}$ is monotonously increasing, the right side is bounded by

$$
\begin{aligned}
(1+\beta)^{-3 / 5} P_{\tilde{T}, \tilde{B}}^{\prime}\left(\tilde{\mu}+\Phi_{C}(\mathbf{x})-W(\mathbf{x})\right) & \\
& \left.\left.\leq\left. C\left(\mid \tilde{\mu}+\Phi_{C}(\mathbf{x})-W(\mathbf{x})\right)\right|_{+} ^{3 / 2}+\exp \left(-\mid \tilde{\mu}+\Phi_{C}(\mathbf{x})-W(\mathbf{x})\right) \mid / \tilde{T}\right)\right)
\end{aligned}
$$

which is in $L^{p}\left(\mathbb{R}^{3}\right)$ for all $1 \leq p<2$.

(iii) This is proved in the same way as Proposition 4.19 (iii) in 8], using Jensen's and Young's inequalities together with (i) and (ii). Note that the Coulomb potential $|\mathbf{x}|^{-1}$ is in $L^{p}\left(\mathbb{R}^{3}\right)_{\text {loc }}$ for $p<3$.

\section{Mean field theory}

For $\rho \in \mathcal{M}$ we define a mean field Hamiltonian by

$$
H_{Z, B, \rho}=[(\mathbf{p}+\mathbf{A}(\mathbf{x})) \cdot \boldsymbol{\sigma}]^{2}+V_{Z, B, \rho}(\mathbf{x})
$$

and a mean field pressure functional by

$$
\mathcal{P}^{\mathrm{mf}}[\rho ; \mu, T, B, Z]=T \operatorname{tr} \ln \left[1+\exp \left\{-\left(H_{Z, B, \rho}-\mu\right) / T\right\}\right]+D(\rho, \rho) .
$$

Note that the first term is equal to

$$
T \ln \operatorname{tr} \exp \left[-\left(\widehat{H}_{Z, B, \rho}-\mu \widehat{N}\right) / T\right]
$$

where $\widehat{H}_{Z, B, \rho}$ is the second quantization of $H_{Z, B, \rho}$. 
By exactly the same methods as in [12, (4.1.10)-(4.1.13), one can show that (3.2) is strictly convex and weakly lower semicontinuous on $\mathcal{M}$ and that the minimizer, $\rho^{\mathrm{mf}}$, is the unique solution of the self-consistent (Hartree) equation

$$
\rho(\mathbf{x})=2\left\langle\mathbf{x}\left|\left(\exp \left\{\left(H_{Z, B, \rho}-\mu\right) / T\right\}+1\right)^{-1}\right| \mathbf{x}\right\rangle .
$$

The right side is here the diagonal of the integral kernel of the trace class opertor $\left(\exp \left\{\left(H_{Z, B, \rho}-\mu\right) / T\right\}+1\right)^{-1}$. Next we introduce the unitary operator

$$
\left(U_{\ell} \psi\right)(\mathbf{x})=\ell^{-3 / 2} \psi\left(\ell^{-1} \mathbf{x}\right)
$$

for $\psi \in L^{2}\left(\mathbb{R}^{3} ; \mathbb{C}^{2}\right)$ with $\ell$ given by (1.13). It transforms the Hamiltonian according to

$$
U_{\ell}^{-1} H_{Z, B, \rho} U_{\ell}=\left(Z \ell^{-1}\right) \tilde{H}_{h, b, \tilde{\rho}}
$$

with

$$
\tilde{H}_{h, b, \tilde{\rho}}=[(h \mathbf{p}+b \mathbf{a}(\mathbf{x})) \cdot \boldsymbol{\sigma}]^{2}+\tilde{V}_{\tilde{\rho}}(\mathbf{x}) .
$$

Here $\mathbf{a}(\mathbf{x})=\frac{1}{2}\left(-x_{2}, x_{1}, 0\right), \tilde{V}_{\tilde{\rho}}$ is defined by (2.19), and

$$
\begin{aligned}
h & =\ell^{-1 / 2} Z^{-1 / 2}=Z^{-1 / 3}(1+\beta)^{1 / 5}, \\
b & =B \ell^{3 / 2} Z^{-1 / 2}=Z^{1 / 3} \beta(1+\beta)^{-3 / 5} .
\end{aligned}
$$

Since the trace is invariant under a unitary transformation we obtain

$$
\begin{array}{r}
T \operatorname{tr} \ln \left(1+\exp \left\{-\left(H_{Z, B, \rho}-\mu\right) / T\right\}\right)= \\
\left(Z \ell^{-1}\right) \tilde{T} \operatorname{tr} \ln \left(1+\exp \left\{-\left(\tilde{H}_{h, b, \tilde{\rho}}-\tilde{\mu}\right) / \tilde{T}\right\}\right),
\end{array}
$$

where $\tilde{T}$ and $\tilde{\mu}$ are given by (2.16). Hence,

$$
\begin{aligned}
\mathcal{P}^{\mathrm{mf}}[\rho ; \mu, T, B, Z] & =Z \ell^{-1} \tilde{T} \operatorname{tr} \ln \left(1+\exp \left\{-\left(\tilde{H}_{h, b, \tilde{\rho}}-\tilde{\mu}\right) / \tilde{T}\right\}\right)+Z^{2} \ell^{-1} D(\tilde{\rho}, \tilde{\rho}) \\
& =Z^{2} \ell^{-1}\left[Z^{-1} \tilde{T} \operatorname{tr} \ln \left(1+\exp \left\{-\left(\tilde{H}_{h, b, \tilde{\rho}}-\tilde{\mu}\right) / \tilde{T}\right\}\right)+D(\tilde{\rho}, \tilde{\rho})\right](3
\end{aligned}
$$

In the next section we study the semiclassical limit $h \rightarrow 0$ of (3.12), which is equivalent to $Z \rightarrow \infty$ with $B / Z^{3} \rightarrow 0$. The minimizer depends on $b$ and $h$, and in order to obtain a semiclassical limit for the minima we need uniform bounds on the minimizers and the corresponding Coulomb potentials. The following lemma is analogous to Lemma 2.2.

Lemma 3.1 (Bounds for mean field minimizer). Let $\tilde{\rho}_{b, h}$ be the minimizer, for fixed $\tilde{\mu}, \tilde{T}$, of

$$
\tilde{\mathcal{P}}^{\operatorname{mf}}[\tilde{\rho} ; \tilde{\mu}, \tilde{T}, b, h]=\left[Z^{-1} \tilde{T} \operatorname{tr} \ln \left(1+\exp \left\{-\left(\tilde{H}_{h, b, \tilde{\rho}}-\tilde{\mu}\right) / \tilde{T}\right\}\right)+D(\tilde{\rho}, \tilde{\rho})\right] .
$$

Then

(i) The potentials $v_{b, h}(\mathbf{x})=\tilde{\rho}_{b, h} *|\mathbf{x}|^{-1}$ are bounded in $L^{6}(\mathbb{R})$ uniformly in $b, h$.

(ii) $\tilde{\rho}_{b, h} \in L^{1}\left(\mathbb{R}^{3}\right)$ and $\left\|\tilde{\rho}_{b, h}\right\|_{1}$ is uniformly bounded in $b, h$. 
(iii) Let $j_{r}=r^{-3} j(\mathbf{x} / r)$ where $0 \leq j \in C_{0}^{\infty}\left(\mathbb{R}^{3}\right)$ satisfies $\int j(\mathbf{x}) d \mathbf{x}=1$. Then for all $R<\infty$ and $p<3$

$$
\left.\left.\int_{|\mathbf{x}| \leq R}\left|v_{b, h}(\mathbf{x})-v_{b, h} *\right| \mathbf{x}\right|^{-1}\right|^{p} d \mathbf{x} \rightarrow 0
$$

uniformly in $b, h$, as $r \rightarrow 0$.

Proof. (i) As in Lemma 2.2 the essential point is that $D\left(\tilde{\rho}_{b, h}, \tilde{\rho}_{b, h}\right)$ is uniformly bounded. In fact,

$$
D\left(\tilde{\rho}_{b, h}, \tilde{\rho}_{b, h}\right) \leq \tilde{\mathcal{P}}^{\mathrm{mf}}\left[\tilde{\rho}_{b, h} ; \tilde{\mu}, \tilde{T}, \beta\right] \leq \tilde{\mathcal{P}}^{\mathrm{mf}}[0 ; \tilde{\mu}, \tilde{T}, \beta]
$$

From the magnetic Lieb-Thirring inequality [8] it follows in the same way as in [12], $(4.1,47)$, that the pressure $\tilde{\mathcal{P}}^{\mathrm{mf}}$ is, up to a constant factor, bounded by the MTF pressure. The uniform bound thus follows in the same way as in Lemma 2.2 (i).

(ii) The Hartree equation for $\tilde{\rho}_{b, h}$ is

$$
\tilde{\rho}_{b, h}(\mathbf{x})=2 Z^{-1}\left\langle\mathbf{x}\left|\left(\exp \left\{\left(\tilde{H}_{h, b, \tilde{\rho}_{b, h}}-\tilde{\mu}\right) / \tilde{T}\right\}+1\right)^{-1}\right| \mathbf{x}\right\rangle .
$$

Since $\tilde{\rho} *|\mathbf{x}|^{-1} \geq 0$, the integral of the right side is bounded by the trace of the operator $2 Z^{-1}\left(\exp \left\{\left(\tilde{H}_{h, b, 0}-\tilde{\mu}\right) / \tilde{T}\right\}+1\right)^{-1}$, which can, again by a magnetic LiebThirring inequality, be bounded by the corresponding semiclassical expression, i.e., $(1+\beta)^{-3 / 5} \int\left(P_{\tilde{T}, \tilde{B}}^{\prime}\left(\tilde{\mu}+\Phi_{C}(\mathbf{x})-W(\mathbf{x})\right) d \mathbf{x}\right.$. This is bounded uniformly in the parameters, by the same estimate as in Lemma 2.2 (ii).

(iii) This is proved in the same way as in Proposition 4.19 in [8], using (i) and (ii).

\section{Semiclassics}

We consider generally the operator

$$
H_{h, b, v}=[(h \mathbf{p}+b \mathbf{a}(\mathbf{x})) \cdot \boldsymbol{\sigma}]^{2}+v(\mathbf{x})
$$

with $h>0, b \in \mathbb{R}, \mathbf{a}(\mathbf{x})=\frac{1}{2}\left(-x_{2}, x_{1}, 0\right)$, and

$$
v(\mathbf{x})=v_{1}(\mathbf{x})+v_{2}(\mathbf{x})
$$

where $v_{1} \in L_{\text {loc }}^{5 / 2}$ and $v_{2}$ is continuous with $v_{2}(\mathbf{x}) \rightarrow \infty$ for $|\mathbf{x}| \rightarrow \infty$. We also impose some further conditions on $v_{1}$ and $v_{2}$ that are described below. In the application to (3.11),

$$
v(\mathbf{x})=\tilde{V}_{\tilde{\rho}}(\mathbf{x})-\tilde{\mu}
$$

and we shall take

$$
v_{1}(\mathbf{x})=-\Phi_{\mathrm{C}}(\mathbf{x})+\tilde{\rho} *|\mathbf{x}|^{-1}
$$

and

$$
v_{2}(\mathbf{x})=W(\mathbf{x})-\tilde{\mu} .
$$


The goal is to find an asymptotic approximation for

$$
P^{\mathrm{Q}}(h, b, v, \tau):=\tau \operatorname{tr} \ln \left(1+\exp \left(-H_{h, b, v}^{1} / \tau\right)\right)
$$

as $h \rightarrow 0$.

The specific conditions for $v_{1}$ and $v_{2}$ are:

1. We assume that outside some compact ball, $B_{R_{0}}=\left\{\mathbf{x}:|\mathbf{x}| \leq R_{0}\right\}, v_{1}$ is subharmonic and the negative part, $\left|v_{1}(\mathbf{x})\right|_{-}:=\left|v_{1}(\mathbf{x})\right|$ for $v_{1}(\mathbf{x})<0$ and 0 otherwise, is continuous and tends to zero at $\infty$. This is fulfilled for (4.4) if $R_{0}>$ $\max _{k}\left|\mathbf{X}_{\mathbf{k}}\right|$. Subharmonicity implies the following property that is convenient for the proof of the upper bound: Let $g \in C_{0}^{\infty}\left(\mathbb{R}^{3}\right)$ with $\int g^{2}(\mathbf{x}) d \mathbf{x}=1$ and define $g_{r}(\mathbf{x})=r^{-3 / 2} g(\mathbf{x} / r)$ for $r>0$. Then

$$
v_{1}(\mathbf{x})-v_{1} * g_{r}^{2}(\mathbf{x}) \geq 0
$$

for $\mathbf{x} \notin B_{R_{0}}$ and $r$ small enough.

2. We assume that $v_{2}$ tends to $\infty$ sufficiently rapidly, so that $e^{-v_{2}(\cdot) / \tau} \in L^{1}\left(\mathbb{R}^{3}\right)$ for all $\tau>0$. (This implies in particular that $e^{-v_{2}(\cdot) / \tau} \in L^{p}\left(\mathbb{R}^{3}\right)$ for all $p \geq 1$.)

3. We assume that $v_{2}$ is sufficiently regular so that following holds: Let $g \in$ $C_{0}^{\infty}\left(\mathbb{R}^{3}\right)$ with $\int g^{2}(\mathbf{x}) d \mathbf{x}=1$ and define $g_{r}(\mathbf{x})=r^{-3 / 2} g(\mathbf{x} / r)$ for $r>0$. Then we assume that there exists a continuous function $v_{2}^{r}$ such that

$$
v_{2}^{r} * g_{r}^{2}=v_{2} .
$$

Moreover, $\lim _{r \rightarrow 0} v_{2}^{r}(\mathbf{x})=v_{2}(\mathbf{x})$ for all $\mathbf{x}$, and $e^{-v_{2}^{r}(\cdot) / \tau} \leq f(\cdot, \tau) \in L^{1}\left(\mathbb{R}^{3}\right)$ for $r$ sufficiently small. The same conditions should be fulfilled for $v_{2, r}:=v_{2} * g_{r}^{2}$.

These conditions on $v_{2}$ are not very restrictive. In fact, Eq. (4.8) has a solution for all $C^{\infty}$ functions $v_{2}$ by [17, Sect. 16.5, and by Fourier transform it easy to check explicitly that the conditions hold, e.g., for all polynomials.

With $P_{T, B}(\mu)$ the pressure of the free electron gas, cf. (1.20) and (2.5), we define

$$
\begin{aligned}
P(w ; h, b, \tau) & :=h^{-3} P_{\tau, h b}(w) \\
& =\tau \sum_{\nu=0}^{\infty} d_{\nu}(h, b) \int_{-\infty}^{\infty} \ln \left[1+\exp \left\{-\left((h p)^{2}+2 \nu h b-w\right) / \tau\right\}\right] d
\end{aligned}
$$

and

$$
P^{\mathrm{scl}}(h, b, v, \tau):=\int P(-v(\mathbf{x}) ; h, b, \tau) d \mathbf{x} .
$$

Then the following holds:

Theorem 4.1 (Semiclassical limit theorem). For fixed $\tau$ and $v$

$$
\lim _{h \rightarrow 0} \frac{P^{\mathrm{Q}}(h, b, v, \tau)}{P^{\mathrm{scl}}(h, b, v, \tau)}=1
$$

uniformly in $b$. 
Proof. We shall make use of convexity of the function

$$
\phi(s)=\tau \ln \left(1+e^{-s / \tau}\right),
$$

which implies the inequality

$$
\phi(s+t) \geq \phi(s)+\phi^{\prime}(s) t .
$$

Moreover, if $L, M$ and $L+M$ are self-adjoint operators then for all $\alpha \geq 1$

$$
\operatorname{tr} \phi(L+M) \leq \operatorname{tr} \phi(L)+\frac{1}{\alpha}[\operatorname{tr} \phi(L+\alpha M)-\operatorname{tr} \phi(L)] .
$$

Another important tool for the proof are the magnetic coherent operators $\Pi(\nu, \mathbf{u}, p)$ with $\nu=0,1,2, \ldots, \mathbf{u} \in \mathbb{R}^{3}$ and $p \in \mathbb{R}$ introduced in [7] and [8]. These operators fulfill the following conditions, cf. Eqs. (3.16)-(3.23) in [8]:

$$
\begin{aligned}
\Pi(\nu, \mathbf{u}, p) & \geq 0 . \\
\sum_{\nu} \iint \Pi(\nu, \mathbf{u}, p) d p d \mathbf{u} & =1, \\
\operatorname{tr} \Pi(\nu, \mathbf{u}, p) & =d_{\nu}(h, b), \\
\operatorname{tr} H_{h, b, v} \Pi(\nu, \mathbf{u}, p) & =d_{\nu}(h, b)\left[\varepsilon_{p, \nu}(h, b)+v * g_{r}^{2}(\mathbf{u})+\frac{h^{2}}{r^{2}} I_{g}\right],
\end{aligned}
$$

with $\varepsilon_{p, \nu}(h, b)=(h p)^{2}+2 b h \nu$ and $I_{g}=\int(\nabla g)^{2}(\mathbf{x}) d \mathbf{x}$. Moreover,

$$
\begin{aligned}
H_{h, b, v}= & \sum_{\nu} \iint\left[\varepsilon_{p, \nu}(h, b)+v_{1}(\mathbf{u})+v_{2}^{r}(\mathbf{u})-\frac{h^{2}}{r^{2}} I_{g}\right] \Pi(\nu, \mathbf{u}, p) d \mathbf{u} d p \\
& +\left(v_{1}-v_{1} * g_{r}^{2}\right) .
\end{aligned}
$$

We also use the following inequalities that are easy consequences of (4.17)-(4.18) and convexity of $\phi$ :

$$
\begin{gathered}
\operatorname{tr} \phi\left(H_{h, b, v}\right) \geq \sum_{\nu} \iint d_{\nu}(h, b) \phi\left(\frac{1}{d_{\nu}(h, b)} \operatorname{tr}\left(H_{h, b, v} \Pi(\nu, \mathbf{u}, p)\right)\right) d \mathbf{u} d p \\
\sum_{\nu} \iint \phi(f(\nu, \mathbf{u}, p)) d \mathbf{u} d p \geq \operatorname{tr} \phi\left(\sum_{\nu} \iint f(\nu, \mathbf{u}, p) \Pi(\nu, \mathbf{u}, p) d \mathbf{u} d p\right)
\end{gathered}
$$

where Eq. (4.22) holds for all sufficiently regular functions $f$ such that both sides are well defined.

The last preparatory step is to note that Eqs. (2.8) and (2.9) imply the following estimates for $P(w ; h, p, \tau)$ :

$$
\begin{aligned}
c\left(h^{-2} b|w|_{+}^{3 / 2}\right. & \left.+h^{-3}|w|_{+}^{5 / 2}\right) \leq P(w ; h, p, \tau) \\
& \leq C\left(h^{-2} b|w|_{+}^{3 / 2}+h^{-3}|w|_{+}^{5 / 2}+e^{-|w| / \tau}\left(h^{-2} b \tau^{3 / 2}+h^{-3} \tau^{5 / 2}\right)\right)
\end{aligned}
$$


and

$$
P^{\prime}(w ; h, p, \tau) \leq C^{\prime}\left(h^{-2} b|w|_{+}^{1 / 2}+h^{-3}|w|_{+}^{3 / 2}+e^{-|w| / \tau}\left(h^{-2} b \tau^{1 / 2}+h^{-3} \tau^{3 / 2}\right)\right) .
$$

Eq. (4.23) implies in particular that for fixed $\tau$ and $v$

$$
P^{\mathrm{scl}}(h, b, v, \tau) \sim\left(h^{-2} b+h^{-3}\right) .
$$

In order to prove (4.12) we thus have to estimate $\tau \ln \left(1+\exp \left(-H_{h, b, v} / \tau\right)\right)$ from above and below by $P^{\mathrm{scl}}(h, b, v, \tau)$ with errors that are small compared to (4.25).

\subsection{Lower bound}

We use (4.19) and begin by writing

$$
\varepsilon_{p, \nu}(h, b)+v * g_{r}^{2}(\mathbf{u})+\frac{h^{2}}{r^{2}} I_{g}=A(\nu, \mathbf{u}, p)+B(\nu, \mathbf{u}, p)
$$

with

$$
\begin{aligned}
& A(\nu, \mathbf{u}, p)=\varepsilon_{p, \nu}(h, b)+v_{1}(\mathbf{u})+v_{2} * g_{r}^{2}(\mathbf{u}), \\
& B(\nu, \mathbf{u}, p)=\frac{h^{2}}{r^{2}} I_{g}+\left(v_{1} * g_{r}^{2}(\mathbf{u})-v_{1}(\mathbf{u})\right) .
\end{aligned}
$$

According to (4.21) and (4.14) we have

$$
\begin{aligned}
P^{\mathrm{Q}}(h, b, v, \tau) & \geq \sum_{\nu} \iint d_{\nu}(h, b) \phi(A+B) d \mathbf{u} d p \\
& \geq \sum_{\nu} \iint d_{\nu}(h, b)\left\{\phi(A)+\phi^{\prime}(A) B\right\} d \mathbf{u} d p .
\end{aligned}
$$

We have to estimate the last term, i.e., the integral over $\phi^{\prime}(A) B$. We use (4.24) and the assumption that $\left|v_{1}(\mathbf{x})\right|_{-} \rightarrow 0$ and $v_{2}(\mathbf{x}) \rightarrow \infty$ if $|\mathbf{x}| \rightarrow \infty$, so $|-v(\mathbf{x})|_{+}=0$ for $\mathbf{x}$ outside some ball $B_{R}=\{\mathbf{x}:|\mathbf{x}| \leq R\}$. Since $v_{2}$ is continuous and $v_{2} * g_{r}^{2}$ therefore bounded on $B_{R}$, uniformly in $r$, the terms involving $I_{g}$ are, after division by $\left(h^{-2} b+h^{-3}\right)$, bounded by

$$
\text { (const.) }\left(\int_{|\mathbf{x}| \leq R}\left|v_{1}(\mathbf{x})\right|^{3 / 2} d \mathbf{x}+1+\int_{|x|>R} \exp \left(-v_{2} * g_{r}^{2}(\mathbf{x}) / \tau\right) d \mathbf{x}\right) h^{2} / r^{2} .
$$

Since $v_{1} \in L_{\text {loc }}^{5 / 2} \subset L_{\text {loc }}^{3 / 2}$ and $\exp \left(-v_{2} * g_{r}^{2} / \tau\right)$ is bounded by an $L^{1}$ function, independent of $r$, (4.27) tends to 0 with $h$ if $r=h^{\delta}, 0<\delta<1$. The terms involving $v_{1} * g_{r}^{2}-v_{1}$ are, up to the factor $\left(h^{-2} b+h^{-3}\right)$, bounded by

$$
\begin{aligned}
& \text { (const.) } \int_{|\mathbf{x}| \leq R}\left(\left|v_{1}(\mathbf{x})\right|^{3 / 2} d \mathbf{x}+1\right)|| v_{1} * g_{r}^{2}(\mathbf{x})-v_{1}(\mathbf{x}) \mid d \mathbf{x} \leq \\
& \text { (const.) }\left(\int_{|\mathbf{x}| \leq R}\left(\left|v_{1}(\mathbf{x})\right|^{5 / 2} d \mathbf{x}+1\right) \mid d \mathbf{x}\right)^{3 / 5}\left(\int_{|\mathbf{x}| \leq R}\left|v_{1} * g_{r}^{2}(\mathbf{x})-v_{1}(\mathbf{x})\right|^{5 / 2} d \mathbf{x}\right)^{2 / 5}
\end{aligned}
$$

which tends to zero with $r$ because $v_{1} \in L_{\text {loc }}^{5 / 2}$. 


\subsection{Upper bound}

Here we use (4.20), (4.15) and (4.22). In addition we need the Lieb-Thirring inequality for a constant magnetic field (see [8]), from which it follows in the same way as in [12, Ex. 1 (4.1, 47), that

$$
\left.P^{\mathrm{Q}}(h, b, v, \tau) \leq \text { (const. }\right) P^{\mathrm{scl}}(h, b, v, \tau) .
$$

Now by (4.20) we can write $H_{h, b, v}^{1}=L+M$ with

$$
L=\sum_{\nu} \iint\left[\varepsilon_{p, \nu}(h, b)+v_{1}(\mathbf{u})+v_{2}^{r}(\mathbf{u})-\frac{h^{2}}{r^{2}} I_{g}\right] \Pi(\nu, \mathbf{u}, p) d \mathbf{u} d p
$$

and

$$
M=\left(v_{1}-v_{1} * g_{r}^{2}\right) .
$$

According to (4.15) we thus have

$$
P^{\mathrm{Q}}(h, b, v, \tau) \leq \operatorname{tr} \phi(L)+\frac{1}{\alpha}[\operatorname{tr} \phi(L+\alpha M)-\operatorname{tr} \phi(L)]
$$

for all $\alpha \geq 1$, and by (4.22) we have

$$
\operatorname{tr} \phi(L) \leq P^{\mathrm{scl}}\left(h, b, v_{1}+v_{2}^{r}-h^{2} I_{g} / r^{2}, \tau\right) .
$$

If $r=h^{\delta}, 0<\delta<1$, then it follows from the properties of $v_{2}$ and the dominated convergence theorem that the right side of (4.31) converges to $P^{\mathrm{scl}}(h, b, v, \tau)$ (in the sense that the ratio tends to 1$)$, if $h \rightarrow 0$.

We thus have to show that it is possible to let $\alpha \rightarrow \infty$ as $r \rightarrow 0$, in such a way that $\operatorname{tr} \phi(L+\alpha M)$ stays bounded by (const.) $\cdot\left(h^{-3}+b h^{-2}\right)$.

Now $L+\alpha M=H_{h, b, v}^{1}+(\alpha-1) L$ and thus

$$
\operatorname{tr} \phi(L+\alpha M)=P^{\mathrm{Q}}\left(h, b, v+(\alpha-1)\left[v_{1}-v_{1} * g_{2}^{r}\right], \tau\right) .
$$

To estimate this we use the inequality (4.29) which gives

$$
\operatorname{tr} \phi(L+\alpha M) \leq \text { (const.) } \int P^{\mathrm{scl}}\left(h, b,-v(\mathbf{x})-(\alpha-1)\left[v_{1}(\mathbf{x})-v_{1} * g_{r}^{2}(\mathbf{x})\right], \tau\right) d \mathbf{x} .
$$

We estimate this further using (4.23) as well as the assumptions on $v_{1}$ and $v_{2}$, which imply that, outside some compact ball $B_{R}$,

$$
\mid-v(\mathbf{x})-(\alpha-1)\left[v_{1}(\mathbf{x})-\left.v_{1} * g_{r}^{2}(\mathbf{x})\right|_{+}=0\right.
$$

and

$$
\mid-v(\mathbf{x})-(\alpha-1)\left[v_{1}(\mathbf{x})-v_{1} * g_{r}^{2}(\mathbf{x})|\geq| v_{2}(x) \mid-(\text { const. })\right.
$$

Now

$$
\int_{|\mathbf{x}| \leq R}\left|v_{1}(\mathbf{x})-v_{1} * g_{r}^{2}(\mathbf{x})\right|^{5 / 2} d x \leq \varphi(r)
$$

for some function $\varphi$ with $\varphi(r) \rightarrow 0$ if $r \rightarrow 0$. If we choose $\alpha=1+\varphi(r)^{-1}$ it follows from (4.23) that $\operatorname{tr} \varphi(L+\alpha M)$ is bounded by (const.) $\cdot\left(h^{-3}+b h^{-2}\right)$ for all sufficiently small $r$. 
In the course of the proof we have shown that (for fixed $\tau$ and $v$ )

$$
\left|P^{\mathrm{Q}}(h, b, v, \tau)-P^{\mathrm{scl}}(h, b, v, \tau)\right|=o\left(h^{-3}+h^{-2} b\right)
$$

and hence, for $h$ and $b$ defined in (3.8), (3.9),

$$
\mid h^{3} P^{\mathrm{Q}}(h, b, v, \tau)-\int P_{\tau, h b}\left(-v(\mathbf{x}) d \mathbf{x} \mid=o(1+h b)=o\left((1+\beta)^{3 / 5}\right) .\right.
$$

Note also that $h^{3}=Z^{-1}(1+\beta)^{3 / 5}, h b=\beta(1+\beta)^{-2 / 5}=\tilde{B}$. We now apply this to compare the MFT functional (1.21) with the mean field functional (3.2), making use of the scalings (2.13) and (3.12). At this point we need to assume that the confining potential $W$ satisfies the regularity conditions stated for $v_{2}$ above. Without further ado we obtain

Corollary 4.1. [Convergence of functionals] If $Z \rightarrow \infty$ while $B / Z^{3} \rightarrow 0$ and $\tilde{\rho}$, $\tilde{\mu}$ and $\tilde{T}$ are fixed, then

$$
\left|Z^{-2} \ell \mathcal{P}^{\mathrm{mf}}[\rho ; \mu, T, B, Z]-\tilde{\mathcal{P}}^{\mathrm{MTF}}[\tilde{\rho} ; \tilde{\mu}, \tilde{T}, \beta]\right| \rightarrow 0 .
$$

For the application to Theorem 1.1, however, we need more than convergence of the functionals for fixed $\tilde{\rho}$, namely the convergence of the minima:*

Corollary 4.2. [Convergence of minima] If $Z \rightarrow \infty$ while $B / Z^{3} \rightarrow 0$ and $\tilde{\mu}, \tilde{T}$ are fixed, then

$$
\left|Z^{-2} \ell P^{\mathrm{mf}}(\mu, T, B, Z)-\tilde{P}^{\mathrm{MTF}}(\tilde{\mu}, \tilde{T}, \beta)\right| \rightarrow 0 .
$$

Proof. Let $\tilde{\rho}_{\beta}$ be the minimizer of $\tilde{\rho} \mapsto \tilde{\mathcal{P}}^{\mathrm{MTF}}[\tilde{\rho} ; \tilde{\mu}, \tilde{T}, \beta]$ for fixed $\tilde{\mu}, \tilde{T}$. Let $\rho_{\beta}$ be the corresponding unscaled density given by (2.15). If $Z \rightarrow \infty$ with $\beta$ fixed (which implies $B / Z^{3} \rightarrow 0$ ) it follows from Corollary 4.1 that

$$
\begin{aligned}
Z^{-2} \ell P^{\mathrm{mf}}(\mu, T, B, Z) & \leq Z^{-2} \ell \mathcal{P}^{\mathrm{mf}}\left[\rho_{\beta} ; \mu, T, B, Z\right] \\
& \rightarrow \mathcal{P}^{\mathrm{MTF}}\left[\tilde{\rho}_{\beta} ; \tilde{\mu}, \tilde{T}, \beta\right]=\tilde{P}^{\mathrm{MTF}}(\tilde{\mu}, \tilde{T}, \beta) .
\end{aligned}
$$

The general condition $B / Z^{3} \rightarrow 0$, however, allows $\beta$ and hence $\tilde{\rho}_{\beta}$ to vary as $Z \rightarrow \infty$ $(h \rightarrow 0)$ so one must check that the error terms in the semiclassical proof are uniform in $\beta$. These terms involve $\int_{|\mathbf{x}| \leq R}\left|v_{1}(x)-v_{1} * g_{r}^{2}(\mathbf{x})\right|^{5 / 2} d \mathbf{x}$ with $v_{1}=-\Phi_{\mathrm{C}}+\tilde{\rho}_{\beta} *|\cdot|^{-1}$ and the required uniformity follows from Lemma 2.2. The converse inequality,

$$
\lim Z^{-2} \ell P^{\mathrm{mf}}(\mu, T, B, Z) \geq \tilde{P}^{\mathrm{MTF}}(\tilde{\mu}, \tilde{T}, \beta),
$$

follows in the same way by noting that the minimizer $\tilde{\rho}_{h, b}$ of the mean field functional enters in in the error terms of the upper bound only through $L^{p}$-norms of $v_{h, b}=$ $\tilde{\rho}_{h, b} *|\cdot|^{-1}$ and $v_{h, b}-v_{h, b} * g_{r}^{2}$ that are uniformly bounded by Lemma 3.1 .

${ }^{*}$ Pointwise convergence of functionals does in general not imply convergence of their infima, even if the functionals are strictly convex and their minimizers lie in a compact set. This can be seen from the following example : Take $\mathcal{F}_{n}(x)=x_{n}+\|x\|^{2}$ on the Hilbert space $\ell^{2}$ of square summable sequences $x=\left(x_{1}, x_{2}, \ldots\right)$. Then $\mathcal{F}_{n}(x) \rightarrow\|x\|^{2}=: \mathcal{F}(x)$ for all $x$ and the infimum of $\mathcal{F}$ is zero. The infimum of $\mathcal{F}_{n}$, on the other hand, is $-1 / 4$. All infima are attained in the weakly compact unit ball in $\ell^{2}$. 


\section{Proof of the QM limit theorem}

In this section we complete the proof of Theorem 1.1. We use the notation explained in the Introduction, in particular $\widehat{H}_{Z, B}$ for the Hamiltonian (1.16) on Fock space and $\widehat{H}_{Z, B, \rho}$ for the second quantization of the mean field Hamiltonian (3.1).

\section{$5.1 \quad$ Upper bound}

We use the inequality 18

$$
\sum_{i<j}^{N} \frac{1}{\left|\mathbf{x}_{i}-\mathbf{x}_{j}\right|} \geq \sum_{i=1}^{N} \int_{\mathbb{R}^{3}} \frac{\rho(\mathbf{x})}{\left|\mathbf{x}-\mathbf{x}_{i}\right|} d \mathbf{x}-D(\rho, \rho)-3,68 \gamma N-\frac{3}{5 \gamma} \int_{\mathbb{R}^{3}} \rho^{5 / 3}(\mathbf{x}) d \mathbf{x}
$$

that holds for all $\gamma>0$ and all $\rho \in \mathcal{M} \cap L^{1}\left(\mathbb{R}^{3}\right) \cap L^{5 / 3}\left(\mathbb{R}^{3}\right)$. Eq. (5.1) implies

$$
\widehat{H}_{Z, B} \geq \widehat{H}_{Z, B, \rho}-3.68 \gamma \widehat{N}-D(\rho, \rho)-C_{\gamma, \rho}
$$

with $C_{\gamma, \rho}=\frac{3}{5 \gamma} \int_{\mathbb{R}^{3}} \rho^{5 / 3}(\mathbf{x}) d \mathbf{x}$. Since $A \geq B$ implies $\operatorname{tr} e^{-A} \leq \operatorname{tr} e^{-B}$, we get an upper bound on the grand canonical pressure:

$$
\begin{aligned}
P^{\mathrm{QM}}(\mu, T, B, Z) & \leq T \ln \left(\operatorname{tr} e^{-\left(\widehat{H}_{Z, B, \rho}-\mu_{\gamma} \widehat{N}\right) / T}\right)+D(\rho, \rho)+C_{\gamma, \rho} \\
& =T \operatorname{tr} \ln \left(1+e^{-\left(H_{Z, B, \rho}-\mu_{\gamma}\right) / T}\right)+D(\rho, \rho)+C_{\gamma, \rho} \\
& =\mathcal{P}^{\mathrm{mf}}\left[\rho ; \mu_{\gamma}, T, B, Z\right]+C_{\gamma, \rho}
\end{aligned}
$$

where $\mu_{\gamma}:=\mu+3.68 \gamma$. We now apply the unitary scaling (3.6) explained in Section 3 and note that

$$
\tilde{\mu_{\gamma}}=\tilde{\mu}+\left(Z^{-1} \ell\right) 3.68 \gamma
$$

and

$$
C_{\gamma, \rho}=\left(Z^{2} \ell^{-1}\right) \frac{3}{5 \gamma Z^{1 / 3} \ell} \int \tilde{\rho}^{5 / 3}(\mathbf{x}) d \mathbf{x}
$$

We choose $\gamma$ as a function of $Z$ and $B$ such that $Z^{-1} \ell \gamma \rightarrow 0$ but $Z^{1 / 3} \ell \gamma \rightarrow \infty$ if $Z \rightarrow \infty$, which is fulfilled as long as

$$
\left[1+\left(B / Z^{4 / 3}\right)\right]^{2 / 5} \ll \gamma \ll Z^{4 / 3}\left[1+\left(B / Z^{4 / 3}\right)\right]^{2 / 5} .
$$

Then, according to (3.12) and Corollary 4.1 we get as $Z \rightarrow \infty$ with $B / Z^{3} \rightarrow 0$ and $\tilde{\mu}, \tilde{T}$ fixed:

$$
\lim \left(Z^{-2} \ell\right) P^{\mathrm{QM}}(\mu, T, B, Z) \leq \tilde{\mathcal{P}}^{\mathrm{MTF}}[\tilde{\rho} ; \tilde{\mu}, \tilde{T}, \beta]
$$

for each $\tilde{\rho} \in \mathcal{M} \cap L^{1}\left(\mathbb{R}^{3}\right) \cap L^{5 / 3}\left(\mathbb{R}^{3}\right)$, in particular for the minimizer $\tilde{\rho}_{\beta}$. Note that $\beta=\infty$ is allowed, c.f. Eq. (2.22), and we can also let $\tilde{\rho}=\tilde{\rho}_{\beta}$ vary for $\beta \rightarrow \infty$, because $\left\|\tilde{\rho}_{\beta}\right\|_{5 / 3}$ is uniformly bounded by Lemma 2.2 (ii). Altogether, by (2.13),

$$
\lim \frac{P^{\mathrm{QM}}(\mu, T, B, Z)}{P^{\mathrm{MTF}}(\mu, T, B, Z)} \leq 1
$$

as $Z \rightarrow \infty$, uniformly in $B$ as long as $B / Z^{3} \rightarrow 0$. 


\subsection{Lower bound}

We use the Peierls-Bogoliubov inequality (12], (2.1.7), (2.1.8)): If $A, B$ and $A+B$ are self-adjoint operators and $F(A)=\ln \operatorname{tr} e^{-A / T}$, then

$$
F(A+B) \geq F(A)-\langle B\rangle_{A}
$$

where

$$
\langle B\rangle_{A}:=\frac{\operatorname{tr}\left(B e^{-A / T}\right)}{\operatorname{tr} e^{-A / T}} .
$$

We use this inequality with $A+B=\widehat{H}_{Z, B}-\mu \widehat{N}$ and $A=\widehat{H}_{Z, B, \rho}-\mu \widehat{N}-D(\rho, \rho)$. Then $B$ is, apart from the constant $D(\rho, \rho)$, the second quantization of

$$
\sum_{i<j}^{N}\left|\mathbf{x}_{i}-\mathbf{x}_{j}\right|^{-1}-\sum_{i=1}^{N} \rho *\left|\mathbf{x}_{i}\right|^{-1}
$$

In terms of the creation and annihilation operators $a_{s}^{*}(\mathbf{x})$ and $a_{s}(\mathbf{x})(s=$ spin component) this can be written

$$
\begin{aligned}
B= & \frac{1}{2} \sum_{s, s^{\prime}} \iint a_{s}^{*}(\mathbf{x}) a_{s^{\prime}}^{*}\left(\mathbf{x}^{\prime}\right)\left|\mathbf{x}-\mathbf{x}^{\prime}\right|^{-1} a_{s^{\prime}}\left(\mathbf{x}^{\prime}\right) a_{s}(\mathbf{x}) d \mathbf{x} d \mathbf{x}^{\prime} \\
& -\int \rho(\mathbf{y}) \sum_{s, s^{\prime}} \int a_{s}^{*}(\mathbf{x}) a_{s}(\mathbf{x})|\mathbf{x}-\mathbf{y}|^{-1} d \mathbf{x} d \mathbf{y}+D(\rho, \rho)
\end{aligned}
$$

We thus get

$$
P^{\mathrm{QM}}(\mu, B, Z, T) \geq T \ln \left(\operatorname{tr} e^{-\left(\widehat{H}_{Z, B, \rho}-\mu \widehat{N}\right) / T}\right)+D(\rho, \rho)-T\langle B\rangle_{A}
$$

We want to show that we can choose $\rho$ such that $\langle B\rangle_{A} \leq 0$.

Since $A$ is the second quantization of a one-particle operator, all expectation values of products of creation and annihilation operators in the state $\langle\cdot\rangle_{A}$ can be written in terms of two point correlations by using Wick's theorem. The expectation value $\langle B\rangle_{A}$ involves terms of the form

$$
\left\langle a_{s}^{*}(\mathbf{x}) a_{s^{\prime}}^{*}\left(\mathbf{x}^{\prime}\right) a_{s^{\prime}}\left(\mathbf{x}^{\prime}\right) a_{s}(\mathbf{x})\right\rangle_{A},
$$

which by can be written

$$
\begin{array}{r}
\left\langle a_{s}^{*}(\mathbf{x}) a_{s}(\mathbf{x})\right\rangle_{A}\left\langle a_{s^{\prime}}^{*}\left(\mathbf{x}^{\prime}\right) a_{s^{\prime}}\left(\mathbf{x}^{\prime}\right)\right\rangle_{A}-\left\langle a_{s}^{*}(\mathbf{x}) a_{s^{\prime}}\left(\mathbf{x}^{\prime}\right)\right\rangle_{A}\left\langle a_{s^{\prime}}^{*}\left(\mathbf{x}^{\prime}\right) a_{s}(\mathbf{x})\right\rangle_{A} \\
=\left\langle a_{s}^{*}(\mathbf{x}) a_{s}(\mathbf{x})\right\rangle_{A}\left\langle a_{s^{\prime}}^{*}\left(\mathbf{x}^{\prime}\right) a_{s^{\prime}}\left(\mathbf{x}^{\prime}\right)\right\rangle_{A}-\left|\left\langle a_{s^{\prime}}^{*}\left(\mathbf{x}^{\prime}\right) a_{s}(\mathbf{x})\right\rangle_{A}\right|^{2} .
\end{array}
$$

If we define

$$
\bar{\rho}(\mathbf{x})=\sum_{s}\left\langle a_{s}^{*}(\mathbf{x}) a_{s}(\mathbf{x})\right\rangle_{A}
$$


then, using (5.16), we can write

$$
\begin{aligned}
\langle B\rangle_{A} & =D(\bar{\rho}, \bar{\rho})-2 D(\rho, \bar{\rho})+D(\rho, \rho)-\frac{1}{2} \sum_{s, s^{\prime}} \iint \frac{\left|\left\langle a_{s^{\prime}}^{*}\left(\mathbf{x}^{\prime}\right) a_{s}(\mathbf{x})\right\rangle_{A}\right|^{2}}{\left|\mathbf{x}-\mathbf{x}^{\prime}\right|} d \mathbf{x} d \mathbf{x}^{\prime} \\
& \leq D(\rho-\bar{\rho}, \rho-\bar{\rho}) .
\end{aligned}
$$

We now choose $\rho=\bar{\rho}$, i.e., we choose $\rho=\rho^{\mathrm{mf}}$ where $\rho^{\mathrm{mf}}$ is the solution of the Hartree equation (3.4). This gives

$$
P^{\mathrm{Q}}(\mu, Z, B, V) \geq P^{\mathrm{mf}}(\mu ; Z, B, T) .
$$

By Corollary 4.2, $P^{\mathrm{mf}}(\mu ; Z, B, T) / P^{\mathrm{MTF}}(\mu ; Z, B, T)$ converges to 1 in the limit considered. Thus

$$
\lim \frac{P^{\mathrm{QM}}(\mu, T, B, Z)}{P^{\mathrm{MTF}}(\mu, T, B, Z)} \geq 1
$$

and the proof of Theorem 1.1 is complete.

\section{Conclusions}

Using magnetic coherent states we have proved a semiclassical limit theorem for a mean field quantum mechanical pressure functional and applied it to derive MFT theory at nonzero temperatures as a limit of quantum statistical mechanics in a certain parameter range. Our result concerns the grand canonical partition function while the corresponding result for the free energy is left as an open problem. Other interesting questions not tackled here concern the extension of the asymptotic ground state classification [19] to temperature and field strength regions beyond those of the present analysis, as well as the thermodynamic limit, where the number of nuclei tends to infinity.

Acknowledgements. We thank Elliott Lieb, Heide Narnhofer, Walter Thirring and Ragnar Sigurdsson for helpful remarks. J.Y. acknowledges the hospitality at the Science Institute of the University of Iceland in Reykjavik where most of this work was carried out.

\section{References}

[1] The Stability of Matter: From Atoms to Stars, Selecta of E.H. Lieb, Ed. by W. Thirring, Springer-Verlag, Berlin etc., 2001.

[2] E.H. Lieb and B. Simon, The Thomas-Fermi Theory of Atoms, Molecules and Solids, Adv. in Math. 23, 22-116 (1977).

[3] E.H. Lieb, Thomas-Fermi and related theories of atoms and molecules, Rev. Mod. Phys. 53, 603-641 (1981); Erratum, Rev. Mod. Phys. 54, 311 (1982). 
[4] I. Fushiki, E. H. Gudmundsson and C. J. Pethick, Surface Structure of Neutron Stars with High Magnetic Fields, Astrophys. J. 342, 958-975 (1989).

[5] I. Fushiki, E. H. Gudmundsson, C. J. Pethick, and J. Yngvason, Matter in a Magnetic Field in the Thomas-Fermi and Related Theories, Ann. Phys. 216, 29-72 (1992).

[6] Ö. E. Rögnvaldsson, I. Fushiki, C.J. Pethick, E.H. Gudmundsson and J. Yngvason, Thomas-Fermi calculations of Atoms and Matter in Magnetic Neutron Stars: Effects of Higher Landau Bands, Astrophys. J., 416, 276 (1993)

[7] J. Yngvason, Thomas-Fermi Theory for Matter in a Magnetic Field as a Limit of Quantum Mechanics, Lett. Math. Phys. 22, 107-117 (1991).

[8] E.H. Lieb, J.P. Solovej, and J. Yngvason: Asymptotics of Heavy Atoms in High Magnetic Fields: II. Semiclassical Regions, Commun. Math. Phys. 161, 77-124 (1994)

[9] V. Ivrii, Asymptotics of the ground state energy of heavy molecules in the strong magnetic field. I, Russian Journal of Math. Phys., 4, 29-74 (1996); II, Russian Journal of Math. Phys., 5, 321-354 (1997)

[10] L. Erdős, J.P. Solovej, Semiclassical eigenvalue estimates for the Pauli operator with strong non-homogeneous magnetic fields: II. Leading order asymptotic estimates, Commun. Math. Phys. 188, 599-656 (1997)

[11] H. Narnhofer, W. Thirring, Asymptotic Exactness of Finite Temperature Thomas-Fermi Theory, Ann. Phys., 134, 128-140 (1981)

[12] W. Thirring, A Course in Mathematical Physics, Vol. 4: Quantum Theory of Large Systems, Springer-Verlag, Wien, 1983.

[13] J. Messer, Temperature Dependent Thomas-Fermi Theory, Springer Lecture Notes in Physics, Vol. 147, Springer, Berlin etc., 1981.

[14] A. Thórólfsson, Ö. E. Rögnvaldsson, J. Yngvason, and E.H. Gudmundsson, Thomas-Fermi Calculations of Atoms and Matter in Magnetic Neutron Stars. II. Finite Temperature Effects, Astrophys. J., 502, 847 -857 (1998).

[15] O.B. Firsov, Calculation of the Interaction Potential of Atoms for Small Nuclear Separation, Sov. Phys.-JETP, 5, 1192-1196, (1957). See also: R. Benguria, The von Weizsäcker and Exchange Corrections in Thomas-Fermi Theory, Ph.D. thesis, Princeton University, 1979 (unpublished).

[16] E.H. Lieb and M. Loss, Analysis, second edition, Amer. Math. Soc. (2001).

[17] L. Hörmander, The Analysis of Linear Partial Differential Operators II, Springer-Verlag, Berlin etc., 1983 
[18] E.H. Lieb and W.E. Thirring, Bound for the Kinetic Energy of Fermions Which Proves the Stability of Matter, Phys. Rev. Lett. 35, 687-689 (1975)

[19] E.H. Lieb, J.P. Solovej, and J. Yngvason: Asymptotics of Heavy Atoms in High Magnetic Fields: I. Lowest Landau Band Regions, Commun. Pure Appl. Math. 47, 513-591 (1994); E.H. Lieb, J. P. Solovej and J. Yngvason, Heavy Atoms in the Strong Magnetic Field of a Neutron Star, Phys. Rev. Lett. 69, 749-752 (1992). 